\title{
Zersplitterte Kommunalparlamente oder Stärkung lokaler Demokratie? Warum die Abschaffung der kommunalen Fünfprozenthürde in Nordrhein-Westfalen ein Fehler war
}

\author{
Jörg Bogumil, Stephan Grohs und Lars Holtkamp
}

Sperrklauseln sollen die Zersplitterung von Parlamenten verhindern, stabile Mehrheiten fördern und so ihre Funktionsfähigkeit gewährleisten. Während diese Zielsetzung auf Bundes- und Landesebene kaum umstritten ist, wurden Sperrklauseln auf kommunaler Ebene in der jüngeren Vergangenheit als unvereinbar mit den Prinzipien der Wahl- und Chancengleichheit erachtet. Eine Folge davon war ihre Abschaffung in fast allen Kommunalwahlgesetzen - auch nach entsprechenden Verfassungsgerichtsurteilen. Welche Konsequenzen sich daraus für die Kommunalparlamente und die politische Steuerungsfähigkeit der Kommunen ergeben, ist bisher kaum empirisch untersucht worden.

Der neuere juristische Diskurs sieht angesichts des Grundsatzes der Wahlgleichheit Sperrklauseln als problematisch und nur in Fällen von deutlichen Funktionsstörungen des kommunalpolitischen Systems als vertretbar an. Was unter einer „Funktionsstörung“ zu verstehen ist, wird jedoch meist einer nur abstrakten Betrachtung unterzogen. So sind auch für Nordrhein-Westfalen, wo die Sperrklausel 1999 abgeschafft wurde, die konkreten Folgen für die Zusammensetzung, die Arbeitsstrukturen und Entscheidungsprozesse in den Kommunalvertretungen unbekannt.

\section{Der juristische Diskurs}

Der Nordrhein-Westfälische Verfassungsgerichtshof hat in Übereinstimmung mit der neueren Verfassungsrechtsprechung und der herrschenden Meinung in der juristischen Literatur festgestellt, dass eine Sperrklausel massiv mit dem Grundsatz der Wahlgleichheit bei der Verhältniswahl konfligiert. Aus Sicht des Gerichts und neuerlich auch des Bundesverfassungsgerichtes verbleibt für Differenzierungen innerhalb der Wahlrechtsgleichheit durch den Gesetzgeber nur ein enger Gestaltungsspielraum, und bei der Prüfung der Zulässigkeit der Differenzierung ist ein strenger Maßstab anzulegen. ${ }^{1}$ Der Gesetzgeber muss sich danach auf eine drohende Funktionsunfähigkeit der Kommunalvertretung als „zwingenden Grund“ berufen. Als „zwingender Grund“2 gilt hier ausdrücklich nicht eine bloße „Schwerfälligkeit der Meinungsbildung “3. Prognosen müssen erstens nachvollziehbar begründet und zweitens auf tatsächliche Entwicklungen gerichtet sein, deren Eintritt ohne Sperrklausel zu erwarten ist beziehungsweise die zum Teil schon eingetreten sind. Bei dieser Beurteilung ist den Besonderheiten eines Landes Rechnung zu tragen.

Dieser neuen Rechtsprechung liegen allerdings bereits einige empirische Annahmen zugrunde, die zu einem signifikanten Wandel der Verfassungsgerichtsurteile und der Positio-

3 NWVerfGH 12/08; NWVBl. 1999, S. 383, S. 385. 
nen in der juristischen Fachliteratur geführt haben. Ursprünglich hatte das Bundesverfassungsgericht gerade mit Hinweis auf die besondere Bedeutung der Großstädte in Nordrhein-Westfalen die Sperrklausel im Kommunalwahlrecht als zulässig eingeordnet. Eine Abschaffung würde in den Großstädten zu einer Zersplitterung der Kommunalparlamente führen und damit die Funktionsfähigkeit der Kommunalvertretungen gefährden, zumal auch der Hauptverwaltungsbeamte vom Rat zu wählen war. ${ }^{4}$ Die grundlegende Veränderung der Rechtsprechung wird überwiegend mit der flächendeckenden Reform der Kommunalverfassungen in den 1990er Jahren in Richtung präsidentieller Systeme begründet. Bereits in seinem 1999 ergangenen Urteil machte der Nordrhein-Westfälische Verfassungsgerichtshof darauf aufmerksam, dass durch diese Reform nun die Hauptverwaltungsbeamten nicht mehr durch die Räte, sondern vom Volk gewählt würden und damit eine wesentliche Begründung aus dem früheren BVerfG-Urteil weggefallen sei. ${ }^{5}$

Im Kern wird damit in der juristischen Literatur und den neueren Verfassungsgerichtsentscheidungen die Position vertreten, dass durch die Direktwahl die „Regierungsbildung“ unabhängig von der Funktionsfähigkeit der Kommunalvertretung gesichert ist. Der direktgewählte Bürgermeister ist aus dieser formalen Perspektive nicht mehr von Hause aus „auf feste Mehrheiten in der Vertretung angewiesen “6, und durch ihn würde eine „politisch aktionsfähige Regierung“ bestehen. In Ausnahmefällen könne die Kommunalaufsicht regulierend eingreifen. Dementsprechend wird überwiegend die Position vertreten, dass Sperrklauseln nach den Kommunalverfassungsreformen in keinem Bundesland mehr für die Kommunalwahlen zu rechtfertigen sind. Dabei werden die sonst überwiegend kritisierten „Absonderlichkeiten der neuen Kommunalverfassung in Nordrhein-Westfalen"7, die insbesondere zu einer relativ starken formalen Stellung des Kommunalparlaments gegenüber dem Bürgermeister beitragen, im Zusammenhang mit der Sperrklausel allerdings ausgeblendet. ${ }^{8}$ Auch die zuvor vom BVerfG hervorgehobene Bedeutung der Ballungsräume in Nordrhein-Westfalen spielt keine Rolle mehr. Es wird demgegenüber festgestellt, dass die Abschaffung der Sperrklauseln in allen Bundesländern „die Funktionsfähigkeit der kommunalen Vertretungsorgane nicht beeinträchtigt hat"), ohne hierfür allerdings einen empirischen Beleg vorzulegen, während von den Befürwortern von Sperrklauseln intensive empirische Untersuchungen zur Untermauerung ihrer Hypothesen angemahnt werden. Derartige Pauschalurteile in der juristischen Literatur berücksichtigen nicht die im neuesten Urteil des BVerfG geforderte „konkrete Prüfung der rechtlichen und tatsächlichen Grundlagen des jeweiligen Landes"10. Ausgeblendet werden vor allem die in der empiri-

4 Vgl. Hans Meyer, Kommunalwahlrecht, in: Thomas Mann / Günter Püttner (Hrsg.), Handbuch der kommunalen Wissenschaft und Praxis, Bd. 1, Berlin 2007, S. 391 - 458, S. 425.

5 Vgl. NW VerfGH 15/98.

6 Hans Meyer, a.a.O. (Fn. 4), S. 427.

7 Hans Herbert von Arnim, Die politische Durchsetzung der Kommunalverfassungsreform der neunziger Jahre, in: DÖV 2002, S. 268.

8 Vgl. ders., Die Unhaltbarkeit der Fünfprozentklausel bei Kommunalwahlen nach der Reform der Kommunalverfassungen, in: Paul Kirchhof/ Moris Lehner / Arndt Raupach / Michael Rodi (Hrsg.), Staaten und Steuern, Heidelberg 2000, S. 453 - 463, S. 459.

9 Markus Krajewski, Kommunalrechtliche Sperrklauseln im föderativen System, in: DÖV 2008, S. 345 - 353; Hans Michael Heinig, Gleichheit trotz(t) Funktionalität. Anmerkungen anlässlich des Urteils des VerfGH NW von 6. Juli 1999 - VerfGH 14/98 und 15/98 - zur Verfassungswidrigkeit der 5 v.H.-Hürde für Kommunalwahlen in NRW, in: NWVBl 2000, S. $121 \mathrm{ff}$.

10 BVerfG, Urteil vom 13. Februar 2008 - 2 BvK 1/07. 
schen Politikwissenschaft erfassten gravierenden Unterschiede der kommunalpolitischen Entscheidungsprozesse im Bundesländervergleich. ${ }^{11}$ Nur aus dieser formalen Perspektive lässt sich dann auch legitimieren, dass die Fünfprozenthürde auf Landesebene zwingend erforderlich ist, weil der Landtag die Regierung bilden muss. Demgegenüber sei durch die Direktwahl des Bürgermeisters bereits „Bestand und Funktion der gemeindlichen Verwaltungsorgane gesichert" 12 .

In der juristischen Literatur wird hervorgehoben, dass die geschilderte neue Rechtsauffassung maßgeblich von einem konkordanzdemokratischen Konzept geprägt sei, in dem die Bürgermeister die kommunale Szenerie dominieren und die Parteipolitisierung deutlich geringer ausgeprägt ist (beziehungsweise sein sollte) als auf der Landes- und Bundesebene. ${ }^{13}$ Gerade aufgrund der Besonderheiten der kommunalen Selbstverwaltung sollten die Sperrklauseln überall aufgehoben werden, um die kommunale „Parteienherrschaft“ einzudämmen. ${ }^{14}$

Die folgenden empirischen Befunde zeigen, dass diese Annahmen auf Nordrhein-Westfalen größtenteils nicht zutreffen, da es sich hier im Vergleich zu allen anderen Bundesländern um viel größere Kommunen mit breitem Aufgabenspektrum handelt und weitere Besonderheiten der Kommunalverfassung anzutreffen sind. Nordrhein-westfälische Kommunen sind aufgrund dieser landesspezifischen Besonderheiten überwiegend konkurrenzdemokratisch strukturiert, und dies lässt erhebliche Probleme für die Funktionsfähigkeit der kommunalen Organe bei einer zunehmenden Fragmentierung der Räte erwarten.

\section{Nordrhein-westfälische Kommunalpolitik im Bundesländervergleich}

Zur Beschreibung der zwischen den Bundesländern sehr stark variierenden kommunalen Entscheidungsstrukturen hat sich in der empirischen Politikwissenschaft das Begriffspaar „Kommunale Konkordanz- und Konkurrenzdemokratie“ durchgesetzt. ${ }^{15}$ Dieser bezieht sich, anders als die klassischen Termini der vergleichenden Regierungslehre (Parlamentarismus, Präsidentialismus etc.), nicht auf die formalen Institutionen. Vielmehr geht es um die Beschreibung von Verhaltens-, Einstellungs- und Einflussmustern der kommunalen Akteure in der Nominierungs-, Wahlkampf-, Wahl- und Regierungsphase. Damit sind also zwei unterschiedliche Typen der repräsentativen Demokratie auf der kommunalen Ebene angesprochen, die sowohl die Kommunalvertretung als auch den seit den 1990er Jahren in allen

11 Vgl. Lars Holtkamp, Parteien und Bürgermeister in der repräsentativen Demokratie - Kommunale Konkordanz- und Konkurrenzdemokratie im Vergleich, in: PVS, 47. Jg. (2006), H. 4, S. 641 - 661; Lars Holtkamp, Kommunale Konkordanz- und Konkurrenzdemokratie - Parteien und Bürgermeister in der repräsentativen Demokratie, Wiesbaden 2008.

12 BayVerfGH 7/04.

13 Vgl. Hans Michael Heinig, a.a.O. (Fn. 9); ders. / Martin Morlok, Konkurrenz belebt das Geschäft! Zur Problematik der 5\%-Klausel im Kommunalwahlrecht, in: Zeitschrift für Gesetzgebung 2000, S. $371-384$.

14 Jörn Ipsen, Die Entwicklung der Kommunalverfassung in Deutschland, in: Thomas Mann / Günter Püttner (Hrsg.), a.a.O. (Fn. 4), S. $565-659$.

15 Jörg Bogumil, Modernisierung lokaler Politik - Kommunale Entscheidungsprozesse im Spannungsfeld zwischen Parteienwettbewerb, Verhandlungszwängen und Ökonomisierung, BadenBaden 2001; Lars Holtkamp, a.a.O. (Fn. 11). 
Bundesländern eingeführten direktgewählten Bürgermeister in die Betrachtungen einbeziehen. Auch auf kommunaler Ebene gilt als konstitutives Unterscheidungsmerkmal, dass in der Konkordanzdemokratie zwischen den Parlamentariern „gütliches Einvernehmen " 16 als Konfliktregelungsmuster überwiegt, während die Konkurrenzdemokratie von Auseinandersetzungen zwischen Mehrheits- und Oppositionsfraktionen geprägt wird. In der Regierungsphase dominiert in der kommunalen Konkordanzdemokratie eine geringe personelle und prozedurale Parteipolitisierung und eine stark ausgeprägte exekutive Führerschaft (dominanter Bürgermeister), in der Konkurrenzdemokratie hingegen eine starke Parteipolitisierung und eine schwächere Stellung des Bürgermeisters. Prozedural wird die parteipolitische Ausrichtung am Umfang konkurrenzdemokratischer Verhaltensmuster erkennbar, der am geschlossenen Abstimmungsverhalten der Fraktionen, der abnehmenden Einstimmigkeit von Ratsbeschlüssen und der klaren Trennung des Rates in Oppositions- und Mehrheitsfraktionen abzulesen ist.

In einer Zusammenschau aller seit 1945 vorgelegten empirischen Untersuchungen zur repräsentativen Demokratie auf kommunaler Ebene konnte gezeigt werden, dass konkordanzdemokratische Muster, die zugleich meist die Vorbilder der normativen juristischen Literatur sind, eher in baden-württembergischen Kommunen dominieren, und in nordrhein-westfälischen Städten konkurrenzdemokratische Konstellationen prägend sind. ${ }^{17}$ Dies kann (neben der Pfadabhängigkeit der Konfliktregelungsmuster) vor allem auf die unterschiedliche Ausprägung zweier unabhängiger Variablen zurückgeführt werden: die durchschnittliche Gemeindegröße und die weiterhin sehr stark variierenden kommunalrechtlichen Rahmenbedingungen im Ländervergleich.

Bezieht man diese allgemeinen empirischen Befunde der lokalen Politikforschung auf die hier vorrangig interessierende Frage der Funktionsfähigkeit der Kommunalparlamente und Verwaltungsorgane, kann als erste These festgehalten werden, dass durch die Einführung der Direktwahl des Bürgermeisters in den überwiegend konkurrenzdemokratisch strukturierten Kommunen in Nordrhein-Westfalen die wesentliche Bedeutung von klaren Mehrheiten in den Kommunalparlamenten nicht abgenommen hat. Immer wenn der direktgewählte Bürgermeister dort über keine verlässliche Mehrheit verfügt, drohen Blockadesituationen und tiefe Eingriffe auch in die Verwaltungsführung des Bürgermeisters, wie sowohl aus vielen Fallstudien als auch aus länderübergreifenden schriftlichen Befragungen der Entscheidungsträger bekannt ist. ${ }^{18}$

Als zweite Hypothese lässt sich formulieren, dass die konkurrenzdemokratische Strukturierung gerade in nordrhein-westfälischen Großstädten den vielen eingezogenen Gruppierungen zwar Vetomacht, aber keine Gestaltungsmacht geben dürfte. Die Beratungen und Verhandlungen sind in der Regel abgeschlossen, wenn die Sitzungsvorlagen in den Rat kommen. Im Rat werden dann nur noch die unterschiedlichen Positionen verkündet, während zuvor in den Ausschussberatungen, aus denen diese Gruppierungen häufig ausgeschlossen sind, durch fachliche Argumente und stärkere konsensuale Orientierung auch

16 Gerhard Lehmbruch, Konkordanzdemokratie, in: Dieter Nohlen (Hrsg.), Wörterbuch Staat und Politik, München 1991, S. $311-316$.

17 Vgl. Lars Holtkamp, a.a.O. (Fn. 11).

18 Vgl. ebenda; ders., Kommunale Haushaltspolitik in den 90er Jahren - Der Wandel von polity, politics und policy, in: Jörg Bogumil (Hrsg.), Kommunale Entscheidungsprozesse im Wandel, Opladen 2002, S. $55-73$. 
kleinere Fraktionen durchaus starken Einfluss nehmen können. ${ }^{19}$ Bei knappen Mehrheiten können kleinere Gruppierungen zwar Anliegen der Verwaltung und der Fraktionen blockieren, aber es ist zu erwarten, dass sie kaum ihre Programmatik durch eigene Ratsanträge umsetzen können.

Zudem nehmen die Komplexität und die zu verarbeitenden Informationen mit der Gemeindegröße stark zu, so dass hier häufig die Grenzen der Ehrenamtlichkeit erreicht sind. Dies kann zum Teil zumindest bei den Fraktionen durch die Finanzierung von zuarbeitendem Fraktionspersonal kompensiert werden. Die nordrhein-westfälischen Großstädte zeichnen sich im Bundesländervergleich durch eine besonders hohe Fraktionsausstattung aus, während die Aufwandsentschädigungen der einzelnen Ratsmitglieder relativ niedrig ausfallen. ${ }^{20}$ Deshalb ist davon auszugehen, dass die Stellung und das Informationsniveau fraktionsloser Ratsmitglieder in den nordrhein-westfälischen Großstädten besonders prekär sind, so dass sie nur schwer gestalterischen Einfluss nehmen können. Auch sie in dauerhafte Koalitionen einzubinden, dürfte schwer fallen, mit der Folge, dass der Anreiz besonders hoch ist, so genannte technische Fraktionen zu bilden, die zwar keine inhaltlichen Gemeinsamkeiten aufweisen, aber den Ratsmitgliedern dieser Gruppierungen doch zum Teil die Möglichkeit geben, an den in Nordrhein-Westfalen besonders ausgeprägten Vorrechten der Fraktionen zu partizipieren. Bei geringem inhaltlichen Zusammenhalt dürften diese Zusammenschlüsse aber ebenfalls kaum dauerhaft in Koalitionen oder dauerhafte Absprachen einbindbar sein. Für die Gründung von technischen Fraktionen ist es vermutlich auch lukrativ, unzufriedene Ratsmitglieder der großen Fraktionen zu umwerben, die bei einem Fraktionsaustritt unter dem Dach einer neuen Fraktion dem Rat weiter angehören.

\section{Zusammensetzung der Kommunalparlamente nach Aufhebung der Sperrklausel}

Die vom Landesverfassungsgericht im aktuellen Urteil herangezogenen Statistiken unterschätzen die Auswirkungen der Aufhebung der Fünfprozenthürde auf die Zusammensetzung der Kommunalparlamente. So wird lediglich auf das Ergebnis einer Kleinen Anfrage von 2007 zu „Ein-Mann-Fraktionen“ rekurriert. ${ }^{21}$ Danach wären durch die Abschaffung der Fünfprozenthürde „nur“ 195 Mandatsträger dieser kleinen Gruppen eingezogen - bei insgesamt 16.838 Ratsmitgliedern in allen kommunalen Vertretungskörperschaften im Lande. Dieser Anteil von gut einem Prozent lässt in der Tat nicht auf eine ernsthafte Gefährdung der Funktionsfähigkeit der Räte schließen.

Anhand von Berechnungen auf der Grundlage von Daten des Landesamtes für Datenverarbeitung und Statistik (LDS) wurde demgegenüber hier der Anteil der Mandatsträger und Fraktionen ermitteln, die 2004 bei einer Fünfprozenthürde nicht in die Parlamente der

19 Vgl. allgemein zur Interaktion von Ausschüssen und Rat Everhard Holtmann, „Das Volk“ als örtlich aktivierte Bürgerschaft - Zur Praxis kommunaler Sachplebiszite, in: Archiv für Kommunalwissenschaften 1999, S. $187-211$.

20 Vgl. Marion Reiser, Berufs- statt Feierabendparlamente? Eine vergleichende Untersuchung der Kommunalparlamente in deutschen Großstädten, in: dies. / Katja Pähle (Hrsg.), Lokale politische Eliten und Fragen der Legitimation, Baden-Baden 2007, S. 45-60; Lars Holtkamp, Professionalisierung der Kommunalpolitik? Empirische und normative Befunde, in: Werner J. Patzelt I Michael Edinger (Hrsg.), Politik als Beruf, PVS-Sonderheft 44, Wiesbaden 2011, S. 103 - 120.

21 LT NRW-Drs. 14/3758. 
kreisfreien Städte und Kreise eingezogen wären. In den kreisfreien Städten sind dies immerhin gut neun Prozent der Mandatsträger, was schon für eine sehr gravierende Veränderung der Zusammensetzung der kommunalen Vertretungskörperschaften spricht. In den Kreistagen ist der Effekt des Verlustes der Sperrklausel deutlich niedriger, was vorwiegend darauf zurückzuführen ist, dass sehr kleine Parteien und lokal ausgerichtete Wählergemeinschaften sich schwerer tun (beziehungsweise ein geringeres Interesse haben), in den großen Flächenkreisen Nordrhein-Westfalens mit einer vollständigen Liste anzutreten. Demgegenüber ist zu erwarten, dass die in unsere Umfrage einbezogenen Kommunen unter 100.000 Einwohner aufgrund der so genannten natürlichen Sperrklausel weniger von diesen Fragmentierungstendenzen betroffen sind als die hier betrachteten kreisfreien Städte. Bei deutlich weniger Ratsmandaten in mittleren und kleineren Kommunen als in einer durchschnittlichen Großstadt müssen Parteien und Gruppierungen schon deutlich höhere Stimmenanteile erlangen, um überhaupt mit einem Sitz vertreten zu sein.

Ein Blick auf die Zusammensetzung dieser Gruppen, die vom Fall der Fünfprozenthürde profitierten, zeigt, dass sowohl in den kreisfreien Städten als auch in den Kreisen vor allem die Wählergemeinschaften zu den größten Gewinnern gehören. Aber auch die rechtsextremen Parteien, die FDP und die PDS ziehen aus der Abschaffung der Sperrklausel klare Vorteile. Sie erhalten hierdurch pro kreisfreie Stadt immerhin ein Ratsmandat mehr.

\begin{tabular}{|c|c|c|c|c|}
\hline \multicolumn{5}{|c|}{$\begin{array}{l}\text { Tabelle 1: Anzahl und Verteilung der Ratsmandate unter der Fünfprozenthürde in den kreisfreien } \\
\text { Städten und Kreisen } 2004\end{array}$} \\
\hline & \multicolumn{2}{|c|}{ Kreisfreie Städte } & \multicolumn{2}{|c|}{ Kreise } \\
\hline Grüne & 3 & $(2 \%)$ & - & \\
\hline FDP & 24 & $(17 \%)$ & - & \\
\hline PDS & 26 & $(18 \%)$ & 9 & $(17 \%)$ \\
\hline REP / DVU / NPD & 21 & $(15 \%)$ & 10 & $(19 \%)$ \\
\hline Wählergemeinschaften & 58 & $(40 \%)$ & 26 & $(48 \%)$ \\
\hline Sonstige & 12 & $(8 \%)$ & 9 & $(17 \%)$ \\
\hline Summe & 144 & & 54 & \\
\hline
\end{tabular}

Insbesondere lohnt ein näherer Blick auf die Wählergemeinschaften, die zuvor gerade in den kreisfreien Städten in Nordrhein-Westfalen kaum Wählerstimmen auf sich vereinigen konnten. 1994 kandidierten in 19 kreisfreien Städten (von 23) jeweils mindestens eine Wählergemeinschaft, in acht davon zwei Wählergemeinschaften. Aber nur in vier Städten konnten sie schließlich Mandate gewinnen. ${ }^{22}$ Demgegenüber kamen 1999 - kurz nach dem Fall der Fünfprozenthürde - bereits 16 Wählergemeinschaften in die Räte kreisfreier Städte. Dies führte offensichtlich zu einer Gründungswelle von Wählergemeinschaften für die Kommunalwahl 2004, die nun auch in kreisfreien Städten ziemlich sicher sein konnten, zumindest ein Ratsmandat zu erringen. Die Zahl der Wählergemeinschaften hat sich im Vergleich zu 1999 mehr als verdoppelt: Im Durchschnitt traten 2004 mehr als zwei Wählergemeinschaften in jeder kreisfreien Stadt an, und schließlich zogen 43 von 50 kan-

22 Vgl. Hiltrud Naßmacher, Die Bedeutung der Kommune und der Kommunalpolitik für den Aufstieg neuer Parteien, in: ZParl, 31. Jg. (2001), H. 1, S. 3 - 18, S. 10. 


\begin{tabular}{|l|c|c|c|}
\hline Tabelle 2: Splitterparteien in den kreisfreien Städten 2004 am Beispiel aller Kandidaturen \\
der Republikaner
\end{tabular}

didierenden Wählergemeinschaften in den Rat ein. Durchschnittlich erhielten diese aber nur unter drei Prozent der Stimmen. Damit sind pro Stadt im Durchschnitt annähernd zwei kleine Wählergemeinschaften vertreten, die häufig aber aufgrund des geringen Wählerzuspruchs keinen Fraktionsstatus erringen konnten.

Ein Blick auf die Tabelle 2 zeigt, wie wenig Stimmen extreme Parteien in Großstädten sammeln müssen (im Verhältnis zu den Wahlberechtigten), wenn eine niedrige Wahlbeteiligung, wie 2004, zu verzeichnen ist. In Köln und Duisburg reichten so beispielsweise unter 0,5 Prozent der Wahlberechtigten für die Republikaner aus, um in den Stadtrat einziehen zu können.

Die Entwicklung der Wählerstimmen in den kreisfreien Städten zeigte für 2004 einen deutlichen Rückgang der beiden großen Volksparteien. Der Konzentrationsgrad des Parteiensystems nahm auch durch den Wegfall der Sperrklausel deutlich ab, und diese Tendenz hat sich zumindest in den kreisfreien Städten bei der Kommunalwahl 2009 durch die Etablierung eines Fünfparteiensystems mit der Partei „Die Linke“ deutlich zugespitzt. Bereits 2004 hatte sich die Anzahl der absoluten Mehrheiten in den kreisfreien Städten deutlich verringert; wenn sich diese Entwicklung, wie zu erwarten, fortsetzt, dürfte die Koalitionsbildung weiter an Bedeutung gewinnen, aber zugleich auch schwerer (mit mehreren Partnern) zu realisieren zu sein.

\section{Auswirkungen der Aufhebung der Sperrklausel auf kommunaler Ebene}

Um zu untersuchen, wie sich die Aufhebung der Sperrklausel auf Arbeitsstrukturen und Entscheidungsprozesse auswirkt, wurde im Januar und Februar 2009 eine schriftliche Umfrage in allen Städten Nordrhein-Westfalens mit über 50.000 Einwohnern durchgeführt. In 
der Summe waren dies 76 Kommunen. Angeschrieben wurden die Ämter für Ratsangelegenheiten beziehungsweise funktional äquivalente Verwaltungseinheiten. Deren Einschätzungen mögen positionsbedingt eine gewisse Verzerrung zugunsten der Präferenz einer reibungslosen und diskursarmen, insoweit an „effizienten“ Entscheidungsprozessen orientierten Verwaltungslogik aufweisen. Angesichts beschränkter Forschungsressourcen gewährleistet deren informierte Perspektive jedoch einen vergleichbaren Blick auf die Arbeitsstrukturen der Räte, der in weiteren Studien um andere Sichtweisen ergänzt werden muss. Auf eine Untersuchung der Landkreise wurde verzichtet, weil in diesen Einheiten deutlich geringere Effekte der Abschaffung der Sperrklausel zu erwarten sind (vgl. Abschnitt 3). Der effektive Rücklauf betrug 80,3 Prozent und weist weder nach Größenklassen noch nach politischer Ausrichtung der Kommunen nennenswerte Verzerrungen auf.

\subsection{Entwicklung und Zusammensetzung der Kommunalparlamente}

In den Kommunalparlamenten kam es nach Aufhebung der Sperrklausel im Jahr 1999 zu einer deutlichen Zunahme der Gruppierungen ohne Fraktionsstatus und von Einzelbewerbern. Ausweislich unserer Erhebung in Städten über 50.000 Einwohner ergibt sich für die vergangenen drei Wahlperioden (1994 bis 1999; 1999 bis 2004; 2004 bis 2009) folgendes Bild: Die Zahl der Ratsfraktionen nach Einführung der Sperrklausel hatte sich um durchschnittlich 1,5 erhöht, die der Gruppierungen und Einzelbewerber ohne Fraktionsstatus

\begin{tabular}{|c|c|c|c|c|c|}
\hline Größenklasse & & 1994-1999 & $1999-2004$ & 2004-2009 & $\begin{array}{c}\text { Veränderung } \\
1994-2004\end{array}$ \\
\hline \multirow{3}{*}{$\begin{array}{l}50.000-100.000 \\
(\mathrm{n}=39)\end{array}$} & Fraktionen & $3,7(3-5)$ & $4,4(3-6)$ & $4,9(3-7)$ & $+1,2$ \\
\hline & $\begin{array}{l}\text { Gruppierungen ohne } \\
\text { Fraktionsstatus }\end{array}$ & $0,1(0-2)$ & $0,5(0-3)$ & $0,7(0-2)$ & $+0,6$ \\
\hline & Gesamt & $3,8(3-5)$ & $4,9(4-8)$ & $5,7(4-9)$ & $+1,9$ \\
\hline \multirow{3}{*}{$\begin{array}{l}100.000-200.000 \\
(\mathrm{n}=11)\end{array}$} & Fraktionen & $3,6(3-4)$ & $4,5(3-6)$ & $5,6(3-7)$ & $+2,0$ \\
\hline & $\begin{array}{l}\text { Gruppierungen ohne } \\
\text { Fraktionsstatus }\end{array}$ & $0,0(0-0)$ & $1,4(0-3)$ & $2,3(0-7)$ & $+2,3$ \\
\hline & Gesamt & $3,6(3-4)$ & $5,9(5-8)$ & $8,0(6-13)$ & $+4,4$ \\
\hline \multirow{3}{*}{$\begin{array}{l}200.000-400.000 \\
(\mathrm{n}=6)\end{array}$} & Fraktionen & $3,1(3-4)$ & $4,0(3-5)$ & $5,0(4-6)$ & $+1,9$ \\
\hline & $\begin{array}{l}\text { Gruppierungen ohne } \\
\text { Fraktionsstatus }\end{array}$ & $0,5(0-2)$ & $1,8(1-4)$ & $2,5(1-6)$ & $+2,0$ \\
\hline & Gesamt & $3,6(3-4)$ & $5,8(3-8)$ & $7,5(5-12)$ & $+3,9$ \\
\hline \multirow{3}{*}{$\begin{array}{l}\text { Über } 400.000 \\
(\mathrm{n}=5)\end{array}$} & Fraktionen & $3,2(3-4)$ & $3,6(3-4)$ & $5,4(5-6)$ & $+2,2$ \\
\hline & $\begin{array}{l}\text { Gruppierungen ohne } \\
\text { Fraktionsstatus }\end{array}$ & $0,0(3-4)$ & $2,4(0-4)$ & $2,0(0-4)$ & $+2,0$ \\
\hline & Gesamt & $3,2(3-4)$ & $6,0(3-8)$ & $7,4(6-9)$ & $+4,2$ \\
\hline \multirow{3}{*}{$\begin{array}{l}\text { Gesamt } \\
(\mathrm{n}=61)\end{array}$} & Fraktionen & $3,6(3-5)$ & $4,3(3-6)$ & $5,1(3-7)$ & $+1,5$ \\
\hline & $\begin{array}{l}\text { Gruppierungen ohne } \\
\text { Fraktionsstatus }\end{array}$ & $0,1(0-2)$ & $0,9(0-4)$ & $1,3(0-7)$ & $+1,2$ \\
\hline & Gesamt & $3,7(3-5)$ & $5,3(3-8)$ & $6,4(4-13)$ & $+2,7$ \\
\hline
\end{tabular}


um 1,3. Während in der Wahlperiode 1994 bis 1999 in über 90 Prozent der Kommunen drei oder vier Fraktionen die Regel waren, keine Fraktionslosen und Gruppierungen vertreten waren, hatten sich diese Werte in der Wahlperiode 2004 bis 2009 auf 28 beziehungsweise 37 Prozent reduziert. Eine detaillierte Betrachtung nach unterschiedlichen Größenklassen macht deutlich, dass die starke Fragmentierung der Räte insbesondere die Städte über 100.000 Einwohner betrifft (vgl. Tabelle 3). Hier nahm die Zahl der Ratsfraktionen sowie der Gruppierungen und Einzelbewerber ohne Fraktionsstatus durchschnittlich um jeweils vier pro Stadt zu. Ausgehend von durchschnittlich unter vier Fraktionen und Gruppierungen 1999 waren 2009 knapp acht Fraktionen und Gruppierungen im Kommunalparlament der typischen nordrhein-westfälischen Großstadt vertreten. Auch nach der Kommunalwahl 2009 gibt es durchschnittlich 7,8 Fraktionen und Gruppierungen in den kreisfreien Städten. Die Werte haben sich also auf einem hohen Niveau stabilisiert.

Dabei zeigt sich, dass der Übergang von Gruppierungen zu Fraktionen fließend ist und dass sich insbesondere in den Großstädten die Gruppierungen beziehungsweise einzelne Ratsmitglieder häufiger zusammenschließen, um Fraktionsstatus zu erwerben. In 40,9 Prozent der Großstädte konnte von 2004 bis 2009 ein Zusammenschluss dieser Gruppen registriert werden. Hinzu kommen noch jene Fraktionen, die durch den Austritt von Ratsmitgliedern aus den etablierten Fraktionen entstanden sind. Diese Entwicklung wird von den Befragten bei den offenen Antwortkategorien besonders problematisiert: „Ratsmitglieder als Einzelpersonen oder Mitglieder einer Gruppe neigen häufig dazu, während der Rats-

\section{Tabelle 4: Fraktionsneugründungen und Intransparenz am Beispiel der Stadt Duisburg}

Der Stadtrat der Stadt Duisburg hat insgesamt 74 Mitglieder. Bei der Kommunalwahl im September 2004 verteilten sie sich wie folgt auf die Parteien:

\begin{tabular}{|c|c|c|c|c|c|c|c|c|c|c|}
\hline & SPD & CDU & Grüne & PDS-OL & FDP & PBP & AMP & REP & DAL & Gesamt \\
\hline 2004 & 28 & 27 & 7 & 4 & 3 & 2 & 1 & 1 & 1 & 74 \\
\hline
\end{tabular}

1. Bereits kurz nach der Wahl ist die DAL in der CDU-Fraktion aufgegangen.

2. Die AMP hatte zunächst mit der FDP eine Fraktion gebildet. Die Duisburger Pro Bürger Partei (PBP) hat sich 2004 von ihrer Bundespartei getrennt und in Bürger Union (BU) 2004 umbenannt. 2005 trennte sich die AMP von der FDP und bildet mit den Vertretern der BU eine gemeinsame Fraktion, der sich auch der REP-Ratsherr anschloss.

3. Die PDS-OL heißt seit Ende 2005 Die Linke. Offene Liste.

4. Im Sommer 2006 kam es zum Bruch der FDP-Fraktion, zwei Mitglieder bilden seither die FDPGruppe, ein FDP-Mitglied bildet zusammen mit einem früheren Mitglied der Bürger Union und der AMP die BL(Bürgerlich-Liberale)/AMP-Fraktion.

5. Ende 2007 hat sich Die Linke. Offene Liste aufgelöst und ist im Kreisverband Duisburg der Partei Die Linke. aufgegangen, die Fraktion trägt seitdem den Namen Die Linke.

6. 2008 sind zwei Ratsmitglieder aus der SPD ausgetreten und haben die Soziale Gruppe Duisburg (SGD) gegründet.

7. Des Weiteren hat sich die Bürger Union 2004 erneut umbenannt in Bürgerunion Duisburg (BU).

8. Ende 2008 ist eine Ratsfrau aus der Fraktion Die Linke. ausgetreten. Sie bleibt bis Ende der Wahlperiode als Fraktionslose Mitglied im Rat.

Somit sah die Sitzverteilung am Ende der Wahlperiode wie folgt aus:

\begin{tabular}{|c|c|c|c|c|c|c|c|c|c|c|}
\hline & CDU & SPD & Grüne & $\begin{array}{c}\text { Die } \\
\text { Linke }\end{array}$ & $\begin{array}{c}\text { BL/ } \\
\text { AMP }\end{array}$ & FDP & BU & SGD & $\begin{array}{c}\text { fraktions- } \\
\text { los }\end{array}$ & Gesamt \\
\hline 2008 & 28 & 26 & 7 & 3 & 3 & 2 & 2 & 2 & 1 & 74 \\
\hline
\end{tabular}


periode die Gruppe zu wechseln oder sich einer Fraktion anzuschließen. Dies hat in unserer Stadt z.B. zur Spaltung einer Fraktion in zwei Gruppen geführt. Später hat sich eine der beiden Gruppen mit einer anderen Gruppe zu einer neuen Fraktion zusammengeschlossen. Auch sonst hat es weitere Fraktionswechsel gegeben. Dadurch kommt nicht nur Unruhe in den Rat, sondern der Wähler kann sich bei diesen Gruppen nie sicher sein, ob sie am Ende noch da sind, wo sie am Anfang der Ratsperiode standen."

Diese Zusammenschlüsse und die damit zum Teil auch zusammenhängenden Bündnisse mit aus den etablierten Fraktionen ausgetretenen Ratsmitgliedern erschweren nicht nur die dauerhaft verlässliche Mehrheitsbildung, sondern führen zu nicht unerheblichen Legitimationsproblemen, weil der Wähler bei diesen Konstellationen kaum in der Lage ist, das Entscheidungsverhalten der von ihm gewählten kleineren Gruppierungen nachzuvollziehen, um bei der nächsten Kommunalwahl gegebenenfalls auch gravierende Abweichungen vom Wahlprogramm sanktionieren zu können. Besonders schwierig dürfte dies sein, wenn sich Gruppierungen zusammenschließen, die keine inhaltlichen Gemeinsamkeiten aufweisen, sondern lediglich den Fraktionsstatus und die damit verbundenen höheren Mittelzuweisungen und erweiterten Rechte anstreben. Wie wenig transparent für die Wählerschaft diese Fraktionsneubildungen sein können, zeigte sich beispielsweise in Duisburg (vgl. Tabelle 4).

Die Mehrheitsverhältnisse wurden in den befragten Kommunen deutlich vom Fragmentierungsgrad der Räte beeinflusst. In über einem Viertel der befragten Räte gab es keine eindeutige Mehrheit (beziehungsweise wechselnde Mehrheiten). Ein hoher Fragmentie-

\begin{tabular}{|c|c|c|c|c|c|}
\hline Mehrheitsverhältnisse & $\begin{array}{l}\text { keine } \\
\text { Fragmen- } \\
\text { tierung } \\
(1-4)\end{array}$ & $\begin{array}{l}\text { leichte } \\
\text { Fragmen- } \\
\text { tierung } \\
(5-6)\end{array}$ & $\begin{array}{l}\text { deutliche } \\
\text { Fragmen- } \\
\text { tierung } \\
(7-8)\end{array}$ & $\begin{array}{l}\text { starke } \\
\text { Fragmen- } \\
\text { tierung } \\
\text { (über 9) } \\
\end{array}$ & Gesamt \\
\hline $\begin{array}{l}\text { keine beziehungsweise stetig } \\
\text { wechselnde Mehrheiten }\end{array}$ & $\begin{array}{c}1 \\
20,0 \% \\
\end{array}$ & $\begin{array}{c}7 \\
23,3 \% \\
\end{array}$ & $\begin{array}{c}7 \\
35,0 \% \\
\end{array}$ & $\begin{array}{c}2 \\
33,3 \% \\
\end{array}$ & $\begin{array}{c}17 \\
27,9 \% \\
\end{array}$ \\
\hline $\begin{array}{l}\text { absolute Mehrheit } \\
\text { einer Fraktion }\end{array}$ & $\begin{array}{c}3 \\
60,0 \% \\
\end{array}$ & $\begin{array}{c}7 \\
23,3 \% \\
\end{array}$ & $\begin{array}{c}1 \\
5,0 \% \\
\end{array}$ & 0 & $\begin{array}{c}11 \\
18,0 \%\end{array}$ \\
\hline $\begin{array}{l}\text { dauerhafte Zusammenarbeit } \\
\text { zwischen Fraktionen (ohne schrift- } \\
\text { liche Vereinbarung) }\end{array}$ & 0 & $\begin{array}{c}7 \\
23,3 \%\end{array}$ & $\begin{array}{c}4 \\
20,0 \%\end{array}$ & $\begin{array}{c}1 \\
16,7 \%\end{array}$ & $\begin{array}{c}12 \\
19,7 \%\end{array}$ \\
\hline $\begin{array}{l}\text { dauerhafte Zusammenarbeit } \\
\text { zwischen Fraktionen und Gruppen } \\
\text { (ohne schriftliche Vereinbarung) }\end{array}$ & 0 & $\begin{array}{c}1 \\
3,3 \%\end{array}$ & $\begin{array}{c}1 \\
5,0 \%\end{array}$ & $\begin{array}{c}2 \\
33,3 \%\end{array}$ & $\begin{array}{c}4 \\
6,6 \%\end{array}$ \\
\hline $\begin{array}{l}\text { dauerhafte Zusammenarbeit mit } \\
\text { Koalitionsvertrag zwischen } \\
\text { Fraktionen }\end{array}$ & $\begin{array}{c}1 \\
20,0 \%\end{array}$ & $\begin{array}{c}8 \\
26,7 \%\end{array}$ & $\begin{array}{c}7 \\
35,0 \%\end{array}$ & $\begin{array}{c}1 \\
16,7 \%\end{array}$ & $\begin{array}{c}17 \\
27,9 \%\end{array}$ \\
\hline Gesamt & 5 & 30 & 20 & 6 & 61 \\
\hline \multicolumn{6}{|c|}{$\begin{array}{l}\text { Anmerkung: Der Fragmentierungsgrad gibt die Anzahl der im Rat vertretenen Fraktionen und sonstigen } \\
\text { Gruppierungen wider. Eine Berechnung üblicher Fragmentierungsindizes war auf Grundlage der vorlie- } \\
\text { genden Daten nicht möglich, da nur die Zahl der Gruppierungen, nicht deren jeweiligen Anteile erho- } \\
\text { ben wurden. Der Fragmentierungsgrad würde zwar unter Berücksichtigung der Sitzanteile weniger deut- } \\
\text { lich ausfallen. Im Weiteren steht jedoch der Einfluss der Kleinstgruppierungen auf die Arbeitsprozesse, } \\
\text { weniger Auswirkungen auf die Mehrheitsbildung im Zentrum; insofern ist der hier verwendete Indikator } \\
\text { für Fragmentierung durchaus der Problemstellung angemessen. } \\
\text { Quelle: Umfrage zur Auswirkung der Sperrklausel 2009; eigene Berechnungen. }\end{array}$} \\
\hline
\end{tabular}


rungsgrad ging häufiger mit uneindeutigen Mehrheiten einher. Eine Zusammenarbeit mit Gruppierungen - sei es mit oder ohne schriftliche Vereinbarungen - fand im Übrigen nur in vier Fällen statt, wie dies bereits vor dem Hintergrund ihrer Binnenstruktur und Strategien zu erwarten war.

\subsection{Kommunale Entscheidungsstrukturen und die Position kleiner Gruppierungen}

Nach Ansicht der meisten juristischen Stellungnahmen sind nach der Direktwahl der Bürgermeister die Mehrheitsverhältnisse in den Kommunalvertretungen unwichtiger geworden. Aus der Perspektive der Befragten trifft diese Einschätzung eindeutig nicht zu. 85 Prozent verneinten die Aussage, dass nach der Einführung der Direktwahl dauerhafte Ratsmehrheiten für die Funktionsfähigkeit der kommunalen Organe unwichtiger geworden sind, deutlich. In Großstädten über 100.000 Einwohner waren es 89 Prozent. Die Mehrheitsverhältnisse sind also weiterhin zentral für die Funktionsfähigkeit der kommunalen Organe in allen betrachteten Gemeindegrößenklassen über 50.000 Einwohner. Wie dies auch in anderen empirischen Untersuchungen hervorgehoben wurde, ist bei generell konkurrenzdemokratischer Strukturierung und starken Eingriffskompetenzen der nordrhein-westfälischen Stadträte auch die Funktionsfähigkeit der kommunalen Verwaltungsspitze nicht unabhängig von den Ratsmehrheiten gegeben. Gerade in den Großstädten gaben die Befragten an, dass bei gegenläufigen Mehrheitsverhältnissen im Stadtrat (die so genannte Kohabitation) häufig Blockadesituationen drohen (80 Prozent). Diese konkurrenzdemokratische Strukturierung der Stadträte in Nordrhein-Westfalen ist zudem daran ablesbar, dass nach Meinung der meisten Befragten die Entscheidungen in Ratssitzungen weitgehend „Formsache“ (93,3 Prozent) sind. Die wesentlichen Vorentscheidungen werden danach in den Fraktionen und Ausschüssen getroffen. Wer in Koalitions- und Ausschussverhandlungen nicht einbezogen wird, hat folglich nur wenige Einflusschancen. Diskussionen im Stadtrat dienen eher der Darstellung unterschiedlicher Positionen, als dass sie die Entscheidungen beeinflussen.

Unter Berücksichtigung dieser konkurrenzdemokratischen Muster stellt sich die Frage, wie die kleineren Gruppierungen versuchen, sich in die kommunalen Beratungs- und Entscheidungsprozesse einzubringen. Dabei ist es bemerkenswert, dass 46,4 Prozent der Befragten feststellten, dass die Gruppierungen an den meisten Ausschusssitzungen, zu denen sie keine Mitglieder entsenden, auch nicht als Zuhörer teilnehmen. Die Gruppen ohne Fraktionsstatus werden ebenfalls nicht in die zentralen Verhandlungen vor der Ratssitzung einbezogen. So gaben knapp 70 Prozent der Befragten an, dass sie bei der Mehrheitsbildung vor der Ratssitzung in der Regel außen vor bleiben. Damit reduzieren sich nicht nur ihre Einflusschancen, sondern es dürfte ihnen auch schwerer fallen, die notwendigen Sachinformationen zu erhalten und zu verarbeiten. Gerade in den Großstädten mit entsprechender Entscheidungskomplexität urteilten die Befragten, dass die Gruppierungen aufgrund der nur gering möglichen Arbeitsteilung inhaltlich häufig überfordert seien (86,7 Prozent über 100.000 Einwohner; 66,7 Prozent unter 100.000 Einwohner) und konstatierten einen deutlichen Informationsvorsprung der Fraktionen (81,8 Prozent über 100.000 Einwohner; 63,7 Prozent unter 100.000 Einwohner). Zu diesem Gesamtbild passt, dass die von den Gruppierungen gestellten Anträge gerade in den Großstadtparlamenten in der Regel folgenlos bleiben (92,9 Prozent über 100.000 Einwohner; 66,3 Prozent unter 100.000 Einwohner).

Gegner der Sperrklausel führen einen Gewinn an demokratischer Qualität und einen höheren Grad an Deliberation durch den Einzug kleiner Gruppierungen in die Kommu- 
nalvertretrungen als positiven Effekt an. Diese Sicht verkennt jedoch die grundlegenden Arbeitsweisen der Kommunalpolitik. Da die wesentlichen Debatten und inhaltlichen Diskussionen in den Ausschüssen geführt werden, trägt die spätere Beteiligung kaum mehr zu einer Bereicherung kommunaler Demokratie oder deliberativer Politikentwicklung bei. Kleinstgruppierungen können häufig die jahrzehntelang eingespielte Arbeitsteilung zwischen Ausschüssen, Bezirksvertretungen und Ratsplenum nicht akzeptieren beziehungsweise durch ihre Binnenstruktur angemessen abbilden und versuchen, im Rat die Debatten der Ausschüsse zu wiederholen (56,3 Prozent über 100.000 Einwohner; 35,7 Prozent unter 100.000 Einwohner), was zu einer deutlichen Verlängerung der Ratssitzungen ohne Veränderung der Beschlussvorlagen führt. Vor diesem Hintergrund verwundert es auch nur wenig, dass die Mehrheit der Befragten diese Gruppierungen prinzipiell als nicht koalitionsfähig einstuft (62,3 Prozent über 100.000 Einwohner; 60,3 Prozent unter 100.000 Einwohner). Ein nicht unbeträchtlicher Teil der Ratsmitglieder ist also nach dem Fall der Fünfprozenthürde kaum in Koalitionen einbindbar. Diese dürften aufgrund des geringen Einbezugs in Verhandlungen und der aus der kaum möglichen Arbeitsteilung resultierenden inhaltlichen Überforderung eher zu gelegentlichen Misstrauensvoten gegenüber der Verwaltung zur Verfügung stehen als für dauerhafte, positiv gestaltende Mehrheiten.

\subsection{Wirkung des Fragmentierungsgrads auf den kommunalen Entscheidungsprozess}

Ein wesentlicher Indikator für den Einfluss des Fragmentierungsgrads auf die kommunalen Entscheidungsprozesse ist die Sitzungszeit. Wie erwartet, zeigt sich ein klarer Zusammenhang der Sitzungszeit mit der Fragmentierung der Kommunalparlamente: Kommunen mit keiner beziehungsweise leichter Fragmentierung haben deutlich kürzere Sitzungszeiten als

\begin{tabular}{|c|c|c|c|c|c|}
\hline $\begin{array}{l}\text { Dauer der } \\
\text { Sitzungen }\end{array}$ & $\begin{array}{c}\text { keine } \\
\text { Fragmentierung } \\
(1-4)\end{array}$ & \begin{tabular}{c|} 
leichte \\
Fragmentierung \\
$(5-6)$
\end{tabular} & \begin{tabular}{c|} 
deutliche \\
Fragmentierung \\
$(7-8)$
\end{tabular} & \begin{tabular}{|c|}
$\begin{array}{c}\text { starke } \\
\text { Fragmentierung } \\
\text { (über 9) }\end{array}$ \\
\end{tabular} & Gesamt \\
\hline deutlich länger & 0 & $\begin{array}{c}4 \\
13,3 \%\end{array}$ & $\begin{array}{c}1 \\
5,3 \%\end{array}$ & 0 & $\begin{array}{c}5 \\
8,3 \%\end{array}$ \\
\hline etwas länger & 0 & $\begin{array}{c}4 \\
13,3 \%\end{array}$ & $\begin{array}{c}7 \\
36,8 \%\end{array}$ & $\begin{array}{c}3 \\
50,0 \%\end{array}$ & $\begin{array}{c}14 \\
23,3 \%\end{array}$ \\
\hline $\begin{array}{l}\text { keine Verände- } \\
\text { rung }\end{array}$ & $\begin{array}{c}4 \\
80,0 \% \\
\end{array}$ & $\begin{array}{c}17 \\
56,7 \% \\
\end{array}$ & $\begin{array}{c}7 \\
36,8 \% \\
\end{array}$ & $\begin{array}{c}2 \\
33,3 \% \\
\end{array}$ & $\begin{array}{c}30 \\
50,0 \% \\
\end{array}$ \\
\hline etwas kürzer & $\begin{array}{c}1 \\
20,0 \%\end{array}$ & $\begin{array}{c}4 \\
13,3 \%\end{array}$ & $\begin{array}{c}1 \\
5,3 \%\end{array}$ & 0 & $\begin{array}{c}6 \\
10,0 \%\end{array}$ \\
\hline deutlich kürzer & 0 & 0 & $\begin{array}{c}1 \\
5,3 \%\end{array}$ & 0 & $\begin{array}{c}1 \\
1,7 \%\end{array}$ \\
\hline keine Angabe & 0 & $\begin{array}{c}1 \\
3,33 \%\end{array}$ & $\begin{array}{c}2 \\
10,5 \%\end{array}$ & $\begin{array}{c}1 \\
16,6 \%\end{array}$ & $\begin{array}{c}4 \\
6,7 \%\end{array}$ \\
\hline Gesamt & 5 & 30 & 19 & 6 & 60 \\
\hline \multicolumn{6}{|c|}{$\begin{array}{l}\text { Anmerkung: Der Fragmentierungsgrad gibt die Anzahl der im Rat vertretenen Fraktionen und sonstige } \\
\text { Gruppierungen wider. } \\
\text { Quelle: Umfrage zur Auswirkung der Sperrklausel 2009; eigene Berechnungen. }\end{array}$} \\
\hline
\end{tabular}




\begin{tabular}{|c|c|c|c|c|c|}
\hline $\begin{array}{l}\text { Zahl der } \\
\text { Anfragen }\end{array}$ & $\begin{array}{c}\text { keine } \\
\text { Fragmentierung } \\
(1-4)\end{array}$ & $\begin{array}{c}\text { leichte } \\
\text { Fragmentierung } \\
(5-6)\end{array}$ & $\begin{array}{c}\text { deutliche } \\
\text { Fragmentierung } \\
(7-8)\end{array}$ & $\begin{array}{c}\text { starke } \\
\begin{array}{c}\text { Fragmentierung } \\
\text { (über 9) }\end{array}\end{array}$ & Gesamt \\
\hline deutlich mehr & 0 & $\begin{array}{c}8 \\
26,7 \% \\
\end{array}$ & $\begin{array}{c}5 \\
27,8 \% \\
\end{array}$ & $\begin{array}{c}2 \\
33,3 \% \\
\end{array}$ & $\begin{array}{c}15 \\
25,4 \% \\
\end{array}$ \\
\hline etwas mehr & $\begin{array}{c}1 \\
20,0 \% \\
\end{array}$ & $\begin{array}{c}4 \\
13,3 \% \\
\end{array}$ & $\begin{array}{c}5 \\
27,8 \% \\
\end{array}$ & $\begin{array}{c}2 \\
33,3 \% \\
\end{array}$ & $\begin{array}{c}12 \\
20,3 \% \\
\end{array}$ \\
\hline nein & $\begin{array}{c}4 \\
80,0 \% \\
\end{array}$ & $\begin{array}{c}15 \\
50,0 \% \\
\end{array}$ & $\begin{array}{c}5 \\
27,8 \% \\
\end{array}$ & $\begin{array}{c}1 \\
16,7 \% \\
\end{array}$ & $\begin{array}{c}25 \\
42,4 \% \\
\end{array}$ \\
\hline etwas weniger & 0 & $\begin{array}{c}1 \\
3,3 \% \\
\end{array}$ & $\begin{array}{c}1 \\
5,6 \% \\
\end{array}$ & 0 & $\begin{array}{c}2 \\
3,4 \% \\
\end{array}$ \\
\hline deutlich weniger & 0 & $\begin{array}{c}1 \\
3,3 \% \\
\end{array}$ & 0 & 0 & $\begin{array}{c}1 \\
1,7 \%\end{array}$ \\
\hline nicht beurteilt & 0 & $\begin{array}{c}1 \\
3,3 \% \\
\end{array}$ & $\begin{array}{c}2 \\
11,1 \% \\
\end{array}$ & $\begin{array}{c}1 \\
16,7 \% \\
\end{array}$ & $\begin{array}{c}4 \\
6,8 \% \\
\end{array}$ \\
\hline Gesamt & 5 & 30 & 18 & 6 & 59 \\
\hline
\end{tabular}

diejenigen mit ausgeprägter Fragmentierung. Dies wird insbesondere im Zeitvergleich deutlich. Betrachtet man die durchschnittlichen Veränderungen in den Sitzungszeiten getrennt nach dem Fragmentierungsgrad, ergibt sich ein noch deutlicheres Bild (vgl. Tabelle 6). Als ein weiterer Indikator für eine stärkere Belastung der Arbeit durch die Fragmentierung der Räte kann die Zahl der Anfragen herangezogen werden. Diese ist in rund 45 Prozent der befragten Städte angestiegen. Vergleicht man die Zunahme der Anfragen nach dem Fragmentierungsgrad, zeigt sich auch hier ein deutlicher Einfluss der Fragmentierung (vgl. Tabelle 7). Bei mehr als der Hälfte der deutlich und stark fragmentierten Kommunen hat sich die Anzahl der Anfragen merklich erhöht, während die weniger oder gar nicht fragmentierten Kommunen kaum Veränderungen wahrnehmen. Angesichts der geringen Einflussmöglichkeiten und des niedrigeren Informationsniveaus ist es sicherlich verständlich, wenn Gruppierungen ohne Fraktionsstatus häufiger auf Anfragen zurückgreifen. Hierdurch dürften nicht unerhebliche administrative Kapazitäten gebunden werden, ohne dass daraus in der Regel andere inhaltliche Beschlüsse resultieren.

\section{Fazit: Eine Sperrklausel ist nötig}

Schon früh hat sich das Bundesverfassungsgericht mit der Verfassungskonformität von Sperrklauseln im nordrhein-westfälischen Kommunalwahlrecht intensiv befasst und verdeutlicht, dass es hierbei juristisch vorwiegend um einen Abwägungsprozess des Grundsatzes der Wahlgleichheit mit dem der Funktionsfähigkeit der Volksvertretungen geht. Diese könne durch eine Zersplitterung des Parlaments gefährdet werden. Im Extremfall sei die Bildung einer mehrheits- und aktionsfähigen Regierung unmöglich: „Klare und ihrer Ver- 
antwortung für das Gesamtwohl bewusste Mehrheiten in einer Volksvertretung sind aber für eine Bewältigung der ihr gestellten Aufgabe unentbehrlich. "23 In diesem Abwägungsprozess kam das BVerfG zunächst für die nordrhein-westfälischen Kommunen zu der Einschätzung, dass die Fünfprozentsperrklausel zulässig ist.

Mit der vorgelegten Untersuchung kann diese Auffassung zur Gefährdung der Funktionsfähigkeit der Kommunalvertretung insbesondere in den Großstädten auf breiter empirischer Basis bestätigt werden. Hier nahm die Zahl der Ratsfraktionen sowie der Gruppierungen und Einzelbewerber ohne Fraktionsstatus durchschnittlich um jeweils vier pro Stadt zu. Ausgehend von durchschnittlich unter vier Fraktionen und Gruppierungen 1999, gab es zehn Jahre später knapp acht Fraktionen und Gruppierungen im Kommunalparlament der typischen nordrhein-westfälischen Großstadt. Diese starke Fragmentierung hat sich mit der Durchsetzung des Fünfparteiensystems auf Bundesebene (im Vergleich noch zu einem Dreiparteiensystem zur Zeit der früheren Urteile des Bundesverfassungsgerichts) auch bei der Kommunalwahl 2009 bestätigt.

In den nordrhein-westfälischen Großstädten wird es durch den Einzug der vielen kleinen Gruppierungen erheblich schwerer, klare Mehrheiten und Koalitionen zu bilden, weil sie auch aufgrund ihrer Binnenstrukturen als kaum koalitionsfähig gelten müssen. Positive gestaltende Mehrheiten und eine aktionsfähige kommunale Regierung werden so deutlich erschwert. Dass die Abschaffung der Sperrklausel die Effizienz und Effektivität kommunalen Regierens in Großstädten gefährdet, ist gerade für Nordrhein-Westfalen ein sehr ernstzunehmender empirischer Befund. Fast die Hälfte der Bürger ist von den Leistungen der politischen Systeme dieser Städte abhängig. Nordrhein-Westfalen stellt die Hälfte aller Großstädte in den alten Bundesländern. Folglich sollten kommunalrechtliche Rahmenbedingungen mit den hier vorzufindenden politischen Entscheidungsmustern kompatibel sein.

Dass die Probleme in den Großstädten besonders zunehmen, ist erstens darauf zurückzuführen, dass aufgrund der hohen Anzahl der Ratsmandate die so genannte natürliche Sperrklausel fast überhaupt nicht greift. ${ }^{24}$ Jede neue Gruppierung, die hier kandidieren möchte, hat weitgehend unabhängig von ihrer Bekanntheit, von Personal und Programmatik fast ein Ratsmandat sicher, was wiederum den Kandidaturanreiz massiv erhöht. Zweitens sind in Großstädten die Parlamente weitgehend konkurrenzdemokratisch strukturiert, mit der Folge, dass starke Fragmentierung zu besonders ausgeprägten Problemen bei der Mehrheitsbildung und Regierungsfähigkeit führt.

Für die nordrhein-westfälischen Großstädte gilt zudem, dass die Kommunalvertretung deutlich mehr Kompetenzen hat als in den meisten anderen Bundesländern. Der Rat kann durch Allzuständigkeit und Rückholrecht in viele Bereiche der Kommunalverwaltung tief eingreifen und die Kompetenzen des hauptamtlichen Oberbürgermeisters durch Zuständigkeitsordnung, die Wahl von Beigeordneten und die Definition ihrer Geschäftsfelder maßgeblich einschränken. Verfügt der Oberbürgermeister im Kommunalparlament nicht über eine eigene, zuverlässige Mehrheit, muss er immer damit rechnen, dass sich fallweise eine „Mobbingkoalition“ gegen ihn bildet. In diesen empirisch gut dokumentierten Fällen blockieren sich Rat und Oberbürgermeister gegenseitig, und die Bildung einer mehrheitsund aktionsfähigen Regierung ist unmöglich, selbst wenn die Kommunalaufsicht bei diesen

23 BVerfGE 51, S. 222.

24 Vgl. Friedrich Pukelsheim / Sebastian Maier / Peter Leutgäb, Zur Vollmandat-Sperrklausel im Kommunalwahlgesetz, in: NWVBl 3/2009, S. 85 - 90. 
Kohabitationskonflikten stark eingreift. ${ }^{25}$ Die Vorstellung, dass die Einführung der Direktwahl des Bürgermeisters bereits „Bestand und Funktion der gemeindlichen Verwaltungsorgane gesichert" 26 hat, trifft empirisch auf die nordrhein-westfälischen Kommunen gerade nicht zu. Die Verwaltung und ihre Führung können nicht weitgehend unabhängig von den konkurrenzdemokratischen Kommunalparlamenten „funktionieren“.

Die rechtlichen und tatsächlichen Grundlagen der Kommunalpolitik in NordrheinWestfalen (starke Ratskompetenzen, Verhältniswahlrecht mit starren Listen, Dominanz extrem großer Kommunen und pfadabhängige konkurrenzdemokratische Entscheidungsmuster) führen also dazu, dass auch nach der Einführung der Direktwahl des Bürgermeisters die Mehrheitsverhältnisse im Kommunalparlament empirisch nicht unwichtiger geworden sind, wie dies in der juristischen Literatur bei einer lediglich formalen Betrachtungsweise erwartet wird und als zentrale Begründung in der stark gewandelten Rechtsprechung zu kommunalen Sperrklauseln fungiert. Vielmehr haben sich die Entscheidungsmuster und die damit grundsätzlich zu erwartenden Probleme bei unklaren Mehrheiten und zersplitterten Kommunalparlamenten in den nordrhein-westfälischen Großstädten kaum verändert.

Zudem führt die Fragmentierung der Räte zu einer geringeren Sitzungs- und Verwaltungseffizienz, ohne dass durch die eingezogenen Gruppierungen neue inhaltliche Impulse in Form von Ratsentscheidungen zu konstatieren sind. Dabei ist es kein Zufall, dass die kleinen Gruppierungen kaum ihrer Programmatik entsprechende inhaltliche Innovationen durchsetzen können. Die Beratungen und Verhandlungen sind in der Regel bei konkurrenzdemokratischen Konstellationen abgeschlossen, wenn die Sitzungsvorlagen in den Stadtrat kommen. Dort werden dann nur noch die unterschiedlichen Positionen verkündet, während zuvor in den Ausschussberatungen, von denen diese Gruppierungen häufig ausgeschlossen sind, durch fachliche Argumente und eher konsensuale Orientierung auch kleinere Fraktionen durchaus starken Einfluss nehmen können. Bei knappen Mehrheiten können kleinere Gruppierungen zwar Anliegen von Verwaltung und Fraktionen im Rat blockieren, aber bei den konkurrenzdemokratischen Entscheidungsmustern in den nordrhein-westfälischen Großstädten kaum ihre Programmatik durch eigene Ratsanträge umsetzen. Zugleich sind sie aufgrund der nur begrenzt möglichen Arbeitsteilung deutlich schlechter informiert als die Fraktionen und haben kaum hauptamtliche Unterstützung für die Ratsarbeit in den eigenen Reihen, so dass sie auch aus diesen Gründen nur schwer inhaltliche Impulse setzen können.

Dieser empirische Befund sollte auch dazu führen, den Grundsatz der Wahlgleichheit, der in der Verfassungsjudikatur eine zentrale Bedeutung hat, zu hinterfragen. Unzweifelhaft ist es wichtig, dass die Wählerstimmen möglichst in gleichem Maße bei der Sitzverteilung im Kommunalparlament Berücksichtigung finden und in den Städten aufgrund der „Nähe“ der kommunalen Ebene eine möglichst enge Beziehung zwischen Repräsentanten und Repräsentierten ermöglicht wird. Allerdings kommt es bei der Beurteilung der InputLegitimität eines Wahlsystems auch darauf an, dass die gewählten Gruppierungen tatsächlich im Parlament die Interessen ihrer Wähler effektiv vertreten können und die Wählerschaft potentiell auch nach einer Wahlperiode beurteilen kann, ob die Gruppierungen und Fraktionen sich bemüht haben, ihre Wahlversprechen einzulösen. Nur durch diese Rück-

25 Vgl. Lars Holtkamp, Governance-Konzepte in der Haushaltskrise, in: Verwaltung und Management 2009, S. $146-159$.

26 BayVerfGH 7/04. 
koppelungsschleife ist in der repräsentativen Demokratie substantielle Repräsentation über die Kontrolle der zeitlich limitierten Macht durch die Wählerschaft realisierbar. ${ }^{27}$ Dies wird in den nordrhein-westfälischen Großstädten durch den Fall der Fünfprozenthürde aber erheblich schwerer. Nicht nur, dass unklare Mehrheitsverhältnisse und damit unklare Verantwortlichkeiten forciert wurden, sondern die hierdurch eingezogenen kleinen Gruppierungen können kaum effektiv das Votum ihrer Wählerschaft in die Kommunalparlamente einbringen. Erschwerend kommt hinzu, dass diese sich nicht selten zu immer wieder neuen Fraktionen zusammenschließen, um an den Zuwendungen partizipieren zu können, während die gemeinsame inhaltliche Basis dieser neuen Fraktionen oft unklar bleibt. Daher ist es der Wählerschaft selbst bei starkem politischen Interesse kaum möglich, die Arbeit der ursprünglich gewählten Gruppierungen zu beurteilen und damit zu kontrollieren.

Eine einheitliche gesetzliche Sperrklausel von 2,5 Prozent im nordrhein-westfälischen Kommunalwahlrecht könnte die Fragmentierung und Zersplitterung der Großstadtparlamente maßgeblich reduzieren, ohne dass in den unteren bis mittleren Gemeindegrößenklassen der Grundsatz der Wahlgleichheit signifikant eingeschränkt würde. In diesen Gemeinden hat die Aufhebung gesetzlicher Sperrklauseln zu weniger Problemen in den Kommunalparlamenten geführt, auch weil hier im stärkeren Maße die natürliche Hürde wirkt. Eine einheitliche Sperrklausel von 2,5 Prozent liegt kaum über dieser natürlichen in kleineren und mittleren Kommunen und würde damit den hier deutlich geringeren Problemen der Fragmentierung angemessen Rechnung tragen. ${ }^{28}$

27 Vgl. Sieglinde Rosenberge / Gilg Seeber, Wählen, Wien 2008.

28 Vgl. Friedrich Pukelsheim / Sebastian Maier / Peter Leutgäb, a.a.O. (Fn. 24).

\title{
Der geliebte Verräter - Zum Einfluss von Spitzenkandidaten auf das Wahlverhalten am Beispiel der saarländischen Landtagswahl 2009
}

\author{
Johannes N. Blumenberg und Manuela S. Kulick
}

Wahlen - und Bundestagswahlen im Besonderen - sind das Ereignis, bei dem die Mehrzahl der Bürger ihre Einstellungen und Meinungen in tatsächliches politisches Verhalten umsetzt. Zugleich ist dies für die meisten auch die einzige aktive Teilnahme an der Politik. Es ist also nicht übertrieben, den Wahlakt als wichtigstes demokratisches Element (nicht nur) im deutschen politischen System zu bezeichnen.

War das Wahlverhalten lange Zeit von Gruppenzugehörigkeit determiniert, so hat sich in den letzten Jahren in der Wahlforschung die Annahme verfestigt, dass immer mehr situative Komponenten, persönliche Vorlieben und Sympathien, beispielsweise die Kandidatenorientierung, bei der tatsächlichen Wahlentscheidung eine große Rolle spielen. Dazu soll hier das kleinste Flächenbundesland, das Saarland, betrachtet werden, wo im Vorfeld der letzten Landtagswahl eine stark auf die Rolle des Kandidaten Oskar Lafontaine fokussierte Medienberichterstattung stattfand, bei der immer wieder auf die tragende Rolle des 